\title{
Teratoma cístico da órbita - estudo clínico patológico: relato de caso
}

\author{
Cystic orbital teratoma - clinicopathologic study: case report
}

\author{
Iluska Fagundes Andrade ${ }^{1}$ \\ Mônica F.Pombo Hilarião ${ }^{2}$ \\ Ricardo Carvalho Rocha ${ }^{3}$ \\ Eduardo Ferrari Marback ${ }^{4}$ \\ Roberto Lorens Marback ${ }^{5}$
}

\begin{tabular}{|c|}
\hline RESUMO \\
\hline Apresentamos um caso de tumor orbitário congênito de grande tamanho, \\
ocorrendoem criançarecém-nascida. A paciente foi submetida a exenteração \\
da órbita e o diagnóstico anatomopatológico foi de teratoma cístico. Os \\
aspectos clínico-patológicos desta rara doença são comentados. \\
\hline
\end{tabular}

Descritores: Neoplasias orbitárias; Teratoma; Teratoma/patologia; Teratoma/congênito; Teratoma/terapia; Relatos de casos [Tipo de publicação]

\section{INTRODUCÃO}

O teratoma cístico da órbita é um tumor congênito benigno raro que causa proptose em recém-nascidos. $\mathrm{O}$ tumor é formado por tecidos normais derivados das três camadas germinativas embrionárias. O primeiro caso de teratoma da órbita foi relatado por Holmes em 1862, sendo posteriormente revisto e classificado como tumor teratóide, pois era formado apenas por duas camadas germinativas ${ }^{(1-2)}$. Em 1876, Bröer e Weigert, relatam o primeiro caso verdadeiro de teratoma da órbita, com documentação da presença de elementos derivados dos três folhetos embrionários ${ }^{(3)}$.

O tumor acomete recém-nascidos sem outros achados associados. Exibe crescimento rápido após o nascimento, causando acentuada proptose e ceratopatia de exposição ${ }^{(2,4-5)}$.

Descreveremos o caso de uma recém-nascida portadora de volumosa tumoração de órbita à esquerda, encaminhado de maternidade local para avaliação diagnóstica e conduta terapêutica.

Trabalho realizado no Departamento de Oftalmologia da Faculdade de Medicina da Universidade Federal da Bahia - UFBA - Salvador (BA) - Brasil.

Aluna do Curso de Especialização em Oftalmologia da Faculdade de Medicina da Universidade Federal da Bahia - UFBA - Salvador (BA) - Brasil.

Aluna do Curso de Especialização em Oftalmologia da Faculdade de Medicina da UFBA - Salvador (BA) - Brasil.

Aluno do Curso de Especialização em Oftalmologia da Faculdade de Medicina da UFBA - Salvador (BA) Brasil.

${ }^{4}$ Doutor, Professor Substituto de Oftalmologia da Faculdade de Medicina da UFBA - Salvador (BA) - Brasil.

5 Professor Titular de Oftalmologia da Faculdade de Medicina da UFBA - Salvador (BA) - Brasil.

Endereço para Correspondência: Eduardo F. Marback Rua Rodrigo Argolo, 89 - Apto. 201 - Salvador (BA) CEP 41940-220

E-mail: eduardomarback@ufba.br

Recebido para publicação em 26.12.2006

Última versão recebida em 25.11.2007

Aprovação em 11.01.2008

\begin{abstract}
APRESENTAÇÃO DO CASO
Paciente do sexo feminino, sete dias de idade, nascida de parto natural a termo, encaminhada com relato de volumosa tumoração em órbita à esquerda presente desde o nascimento. A genitora referiu acompanhamento prénatal completo sem intercorrências (seis consultas e três ultra-sonografias), não havendo identificação do tumor às ultra-sonografias.

Ao exame notava-se tumor muito volumoso em órbita esquerda, impedindo oclusão de fenda palpebral e exibindo ulceração e pontos hemorrágicos em conjuntiva (Figura 1A). O olho direito era normal. Foi solicitada tomografia computadorizada (TC) das órbitas para programação cirúrgica. Face às dificuldades em obter a TC e conseguir reserva de vaga em unidade de terapia intensiva (UTI) neonatal, a cirurgia só foi realizada 15 dias após a primeira consulta. A TC mostrava alargamento da cavidade orbitária esquerda, que estava preenchida por tumor com densidade heterogênea, compatível com partes moles, exibindo áreas de
\end{abstract}


calcificação e císticas, sem aparente extensão intracraniana (Figura 1B).

A suspeita diagnóstica foi de teratoma cístico de órbita. A paciente foi submetida a exenteração parcial da órbita, poupando pele palpebral, sendo necessária hemotransfusão péroperatória e cuidados pós-operatórios em UTI neonatal. Em relação ao exame inicial, o tumor exibia no dia da cirurgia, acentuado aumento de volume com perfuração de globo ocular e extrusão de seu conteúdo (Figura 1C). Evoluiu com boa cicatrização por segunda intenção da cavidade e sem sinal de recidiva após 22 meses de acompanhamento (Figura 1D). Apesar de ter sido encaminhada para adaptação de prótese, os pais optaram por não fazê-lo.

Macroscopicamente a peça cirúrgica media 8,0x6,5×5,0 cm, apresentava coloração castanho-amarelada e superfície irregular. À superfície de corte, notava-se tecido esbranquiçado com partes pigmentadas e áreas císticas preenchidas por líquido claro, não sendo possível identificar estruturas oculares (Figura 2).
À microscopia havia neoplasia benigna constituída por elementos derivados dos três folhetos embrionários. Do ectoderma havia tecido nervoso. Do mesoderma: tecido adiposo, músculo liso e cartilagem. Do endoderma: cistos revestidos por epitélio intestinal e respiratório (Figura 2).

\section{DISCUSSÃO}

O teratoma é um tumor embrionário derivado das três camadas germinativas. Usualmente há predomínio de componentes ectodérmicos e mesodérmicos. Os elementos endodérmicos freqüentemente estão ausentes ou são difíceis de detectar ${ }^{(4,6)}$. Nestes casos, a denominação utilizada é a de tumor teratóide $^{(4)}$. As camadas germinativas surgem de células pluripotentes e, portanto, estão presentes principalmente nas gônadas, mais raramente podem ser encontradas nos restos de células primitivas seqüestrados em outras áreas ${ }^{(7)}$. Raramente, pode haver transformação maligna ${ }^{(6,8)}$.
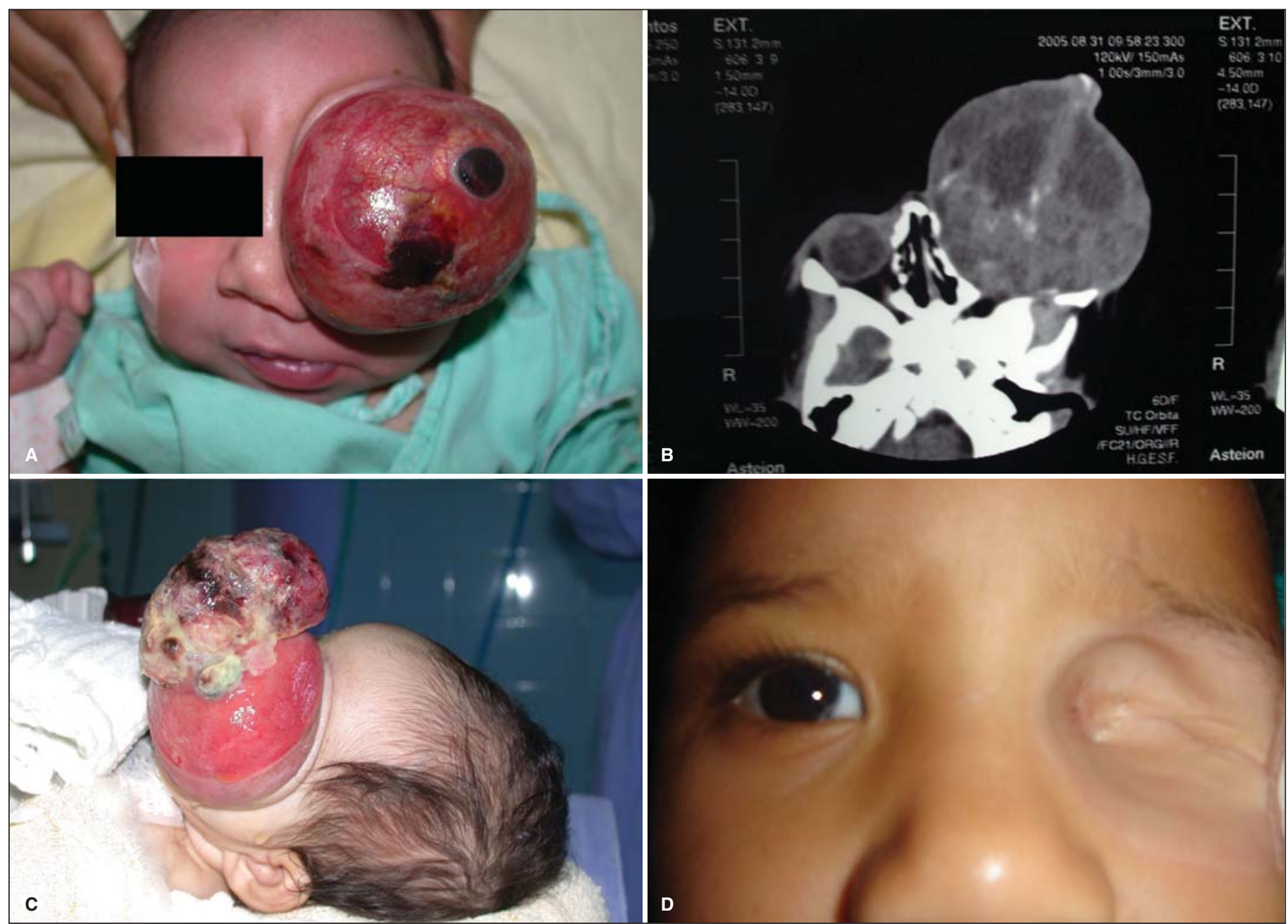

Figura 1 - A) Foto clínica à apresentação: tumor orbitário volumoso com ulceração conjuntival; B) Tomografia computadorizada: lesão preenchendo toda a cavidade, com alargamento do arcabouço ósseo. O tumor apresenta calcificações e áreas císticas em seu interior; C) Aspecto clínico no pré-operatório imediato com perfuração do globo ocular; D) Aspecto após 22 meses da cirurgia. 

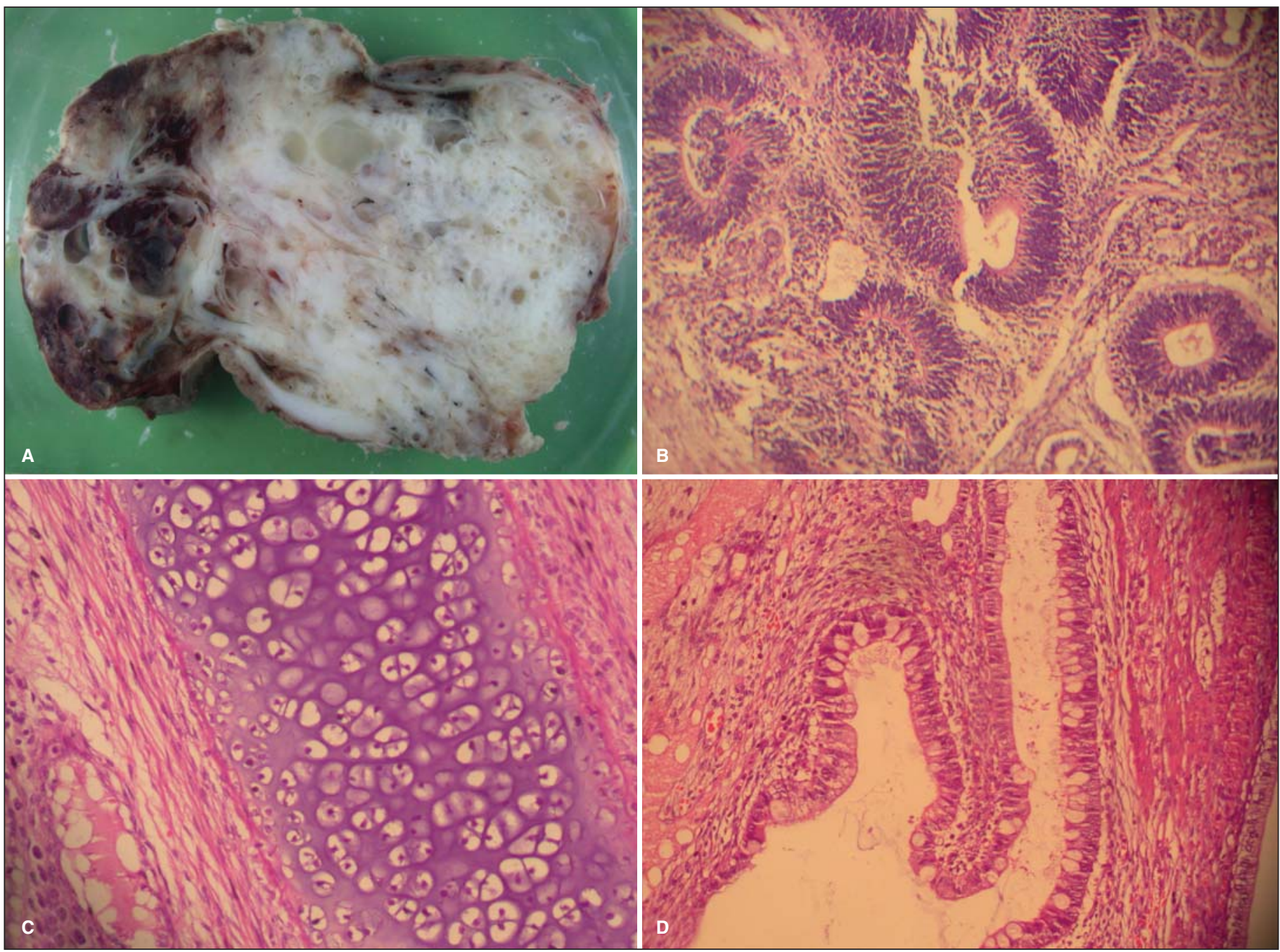

Figura 2 - A) Macroscopia: tumor com múltiplos cistos e estruturas oculares não individualizadas; B) Microscopia: tecido neuro-ectodérmico, simulando tubo neural (hematoxilina e eosina); C) Microscopia: tecido cartilaginoso - origem mesodérmica (hematoxilina e eosina); D) Microscopia: cisto revestido por epitélio do tipo intestinal - origem endodérmica (hematoxilina e eosina)

As células pluripotentes podem se diferenciar em várias linhagens germinativas ${ }^{(7)}$. O ectoderma superficial dá origem ao epitélio escamoso estratificado, folículos pilosos e glândulas sudoríparas. O neuro-ectoderma inclui o tubo neural primitivo, plexo coróide e gânglios. O mesoderma é representado por músculo, osso, cartilagem e gordura. O endoderma pode originar tecido gastrointestinal e epitélio colunar pseudoestratificado do tipo respiratório ${ }^{(7)}$.

A revisão de literatura mostra que o teratoma de órbita predomina em olho esquerdo $(60 \%)$ e a relação entre sexo feminino e masculino é de $2: 1$. Sua característica típica é o rápido crescimento após o nascimento, por isso, tumores não tratados por um período de meses podem alcançar grandes dimensões $^{(2,4,6)}$. Com a expansão da massa pode ocorrer compressão do globo ocular e nervo óptico, ceratopatia de exposição e eventual perfuração, como no nosso caso $^{(2,4,6)}$.

As características mais freqüentemente encontradas no teratoma orbitário são acentuada proptose unilateral em recém- nascidos, distensão das pálpebras e aumento da fenda palpebral, ausência de comunicação direta entre as cavidades císticas e a cavidade intra-craniana, desenvolvimento normal do olho com freqüentes mudanças degenerativas secundárias ao crescimento do tumor, aumento da cavidade orbitária cerca de 2 a 3 vezes o tamanho normal e transiluminação de parte ou de todo o tumor ${ }^{(6,9)}$.

No passado a exenteração da órbita em crianças com teratoma estava associada a uma alta taxa de mortalidade. Atualmente com o avanço das técnicas anestésicas, cirúrgicas e de cuidado pós-operatório em UTI neonatal, houve diminuição da mortalidade destes pacientes ${ }^{(5)}$. $\mathrm{O}$ tratamento cirúrgico quase sempre é efetivo, conseguindo a exérese completa do tumor. Entretanto, mesmo nos casos de teratomas orbitários pequenos nos quais é possível a ressecção isolada do tumor, raramente a função visual pode ser preservada, devido às alterações secundárias no globo ocular e nervo óptico ${ }^{(6,10)}$. Não encontramos, na literatura pesquisada, referência a rea- 
bilitação tardia dos pacientes submetidos a exenteração por teratoma orbitário. Nossa paciente foi encaminhada para adaptação de prótese, porém por opção dos pais, permanecia sem prótese quase dois anos após a cirurgia.

Merecem destaque em nosso caso, o acentuado volume da tumoração, e o fato da mesma não ter sido observada às ultrasonografias pré-natais. Vale lembrar ainda que tais recémnascidos apresentam volemia em torno de $280 \mathrm{ml}$. Com volemia tão pequena, é necessário dispor de sangue para reposição na programação cirúrgica. No caso em questão, conseguimos minimizar o sangramento durante o ato cirúrgico através ligadura do pedículo do tumor no vértice da órbita, utilizando faixa umbilical e cauterização com bisturi elétrico.

Dentre os 7.500 casos submetidos a estudo no Laboratório de Patologia Ocular do Serviço de Oftalmologia do Hospital Professor Edgar Santos até o momento, este é segundo caso de teratoma cístico da órbita, o que enfatiza sua raridade.

\section{ABSTRACT}

We report on a case of an congenital orbital tumor of impressive size, occurring in a newborn. The patient underwent orbital exenteration with a histopathologic diagnosis of cystic teratoma. The clinicopathological aspects of such a rare disease are commented.
Keywords: Orbital neoplasms; Teratoma; Teratoma/pathology; Teratoma/congenital; Teratoma/therapy; Case reports [Publication type]

\section{REFERÊNCIAS}

1. Holmes T. Congenital tumour removed from the orbit. Trans Pathol Soc Lond. 1862-3;14:248.

2. Levin ML, Leone CR Jr, Kincaid MC. Congenital orbital teratomas. Am J Ophthalmol. 1986;102(4):476-81.

3. Broer WC, Weigert C. Teratoma orbitae congenitum. Virchows Arch Pathol Anat. 1876;67:518-22.

4. Weiss AH, Greenwald MJ, Margo CE, Myers W. Primary and secondary orbital teratomas. J Pediatr Ophthalmol Strabismus. 1989;26(1):44-9.

5. Ferry AP. Teratoma of the orbit: a report of two cases. Surv Ophthalmol. 1965;10(5):434-42.

6. Garrity JA, Henderson JW. Orbital teratoma. In: Garrity JA, Henderson JW, Cameron JD. Henderson's orbital tumors. $4^{\text {th }}$ ed. Philadelphia: Lippincott Williams \& Wilkins; 2007. p.44.

7. Robins SL, Kumar V, Cotran RS, Schoen FJ. Neoplasias. In: Robins SL, Cotran RS, Kumar V. Patologia estrutural e funcional. $5^{\underline{a}}$ ed. Rio de Janeiro: Guanabara Koogan; 1996. p.215.

8. Mahesh L, Krishnakumar S, Subramanian N, Babu K, Biswas J. Malignant teratoma of the orbit: a clinicopathological study of a case. Orbit. 2003;22(4): 305-9.

9. Hoyt WF, Joe S. Congenital teratoid cyst of the orbit. A case report and review of the literature. Arch Ophthalmol. 1962;68:196-201.

10. Gnanaraj L, Skibell BC, Coret-Simon J, Halliday W, Forrest C, DeAngelis DD. Massive congenital orbital teratoma. Ophthal Plast Reconstr Surg. 2005; 21(6):445-7. 